\title{
Ueber die sog. Xantholeukophoren beim Laubfrosch.
}

\author{
Von \\ Prof. Dr. W. J. Schmidt, Bonn, Zoologisches Institut. \\ Hierzu Tafel IV.
}

Historisches.

Der Laubfrosch (Hyla arborea) bietet wohl unter allen einheimischen Amphibien den ausgeprägtesten Farbenwechsel dar. Während die Physiologie des Chromatophorenspiels vor allem durch die Untersuchungen von $\mathrm{Bi}$ e $\mathrm{d}$ e r ma $\mathrm{n} \mathrm{n}$ in wesentlichen Zügen klar gestellt ist, herrschen hinsichtlich einer morphologischen Frage nämlich der Beschaffenheit der sogenannten Xanthole uko$\mathrm{p}$ h o r e n noch gegensätzliche Meinungen, wenigstens, wenn man sich an die Originalarbeiten hält. Die ein€n Autoren nehmen an, daß an den grünen Hautstellen $G$ uanin und Li pochrom in ein und derselben $Z$ elle nebeneinander und $z$ war in bestimmter Anordnung vorkommen; diese Zellen werden jetzt gewöhnlich nach dem Vorgang von $G$ a u p p als Xantholeukophoren bezeichnet. Die anderen dagegen, vertreten den Standpunkt, daß diese $\mathrm{be}$ ide $\mathbf{n}$ am Farbenwechsel wesentlich beteiligten $\mathrm{Sub}$ stanzen jede für sich in besonderen Zellen liegen, das gelbe Lipochrom in den $\mathrm{Xanthophoren,} \mathrm{die}$ man auch als Lipophoren bezeichnen könnte (vgl. W. J. S c h m i d t 1917), die Guaninmassen in tiefer in der Haut eingebetteten Zellen, den $\mathrm{L}$ e u k o phoren (Guanophoren). Die zusammenfassenden Darstellungen gehen mit Ausnahme von van. Ry n berk nicht 
näher auf diese Streitfrage ein, betrachten sie vielmehr als im Sinne der Existenz von Xantholeukophoren entschieden, so $G$ a $\mathrm{p} \mathrm{p}$, Fuchs, Hempelmann. Die nachfolgende Untersuchung wird aber dartun, daß diese Auffassung ganz bestimmt verkehrt ist und diejenigen Forscher im Recht sind, welche Guanin und Lipochrom auch an den grünen Hautstellen jedes für sich in besonderen Zellen vorkommen lassen. $D$ as Verdienst, diese Verhältnisse $z$ um erstenmal über jeden $Z$ weifel klargestelltzuhaben, gebührt Ficalbi.

Gehen wir zunächst auf die Angaben der beiden Richtungen näher ein. $F$ ü $r$ die Existenz von Xantholeukophoren haben sich

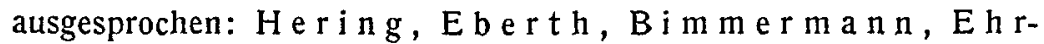
mann, Biedermann, Sied lecki und im AnschluB an deren Originalarbeiten $G$ a $\mathrm{pp}, \mathrm{He} \mathrm{mpel} \mathrm{m}$ a n $\mathrm{n}$ und $\mathrm{Fuchs}$.

H e r ing (1869, S. 50 f.) sagt, daß die grüne Färbung der Haut bei $R$ ana esculenta und $H y l a$ arborea hauptsächlich durch die Anwesenheit von zweierlei Pigmentzellen bedingt werde, und zwar fänden sich an den grünen Hautstellen ziemlich dicht unterhalb der Epidermis unregelmäBig polygonale, dicht gelagerte $\mathrm{g}$ e $\mathrm{b}$ e $\mathrm{Z}$ ell e $\mathrm{n}$ und darunter sternförmige mit schwarzbraunem Pigment. Der Inhalt der gelben Zellen das sind unsere Xantholeukophoren - bestehe aus einem in Alkohol und Aether löslichen gelben Fett u n d aus stark lichtbrechenden prismatischen farblosen Körnchen. Wird das Fett den gelben Zellen entzogen, berichtet He ring weiter, so bleiben die Körnchen zurück; die Zelle nimmt dann ein grauweißes Aussehen an und stellt sich ähnlich dar wie die an den ungefärbten Stellen der Haut vorkommenden sternförmigen Zellen, weiche schon in frischem Zustande einen gleichen farblosen, körnigen, das Licht stark reflektierenden Inhalt zeigen. Aehnliche sternförmige Zellen finden sich auch an den rein gelben Stellen der Haut, aber dieselben enthalten außer den eben erwähnten Körnchen auch noch jenen gelben, durch Aether und Alkohol ausziehbaren Farbstoff. Durch schwache Kalilösung werden die prismatischen Körnchen in den gelben Zellen gelöst, während der gelbe Farbstoff in Tropfenform zurückbleibt. Bei Nahrungsentziehung schwindet dieses Fett teilweise.

E b e r th (1869, S. 14 f.) findet bei $\mathrm{H} \mathrm{y} \mathrm{l}$ a an den grünen Hautstellen unter dem zarten Grenzsaum der Kutis eine nach Art eines platten Epithels angeordnete Lage rundlicher und polygonaler Zellen, deren Protoplasma außer dem Kern eine Menge punktförmiger $\mathrm{g} r$ a u e $\mathrm{K}$ ö $\mathrm{r} \mathrm{n} \mathrm{h}$ e $\mathrm{n}$ enthält, diemit goldgelben Tröpfchen untermengt si n d. Die gelben Tröpfchen lösen sich in Alkohol, die neben ihnen befindlichen interferierenden Körnchen dagegen leiden nicht durch Kochen mit Alkohol und Chloroform, werden dagegen rasch durch Kalilauge und Sàlzsäure zerstört. Sollten diese Zellen ihre Form verändern können, so sind 
diese Kontraktionen nach $E$ b e $t_{h} \mathrm{~h}$ auBerordentlich schwach und erfolgen sehr langsam.

B i m m e rma n n (1878, S. 27), der mit $R$ a n a e s culen t a, aber auch mit $\mathrm{Hyla}$ arbeitete, unterscheidet neben schwarzem Pigment in Zellen und dunkelbraunem, frei im Gewebe zerstreuten (? Sch.) ein "h e l I e s Pigmentin Zellenmitgrobgranulärem Inhal t". Diese Zellen sind an den Seiten des Körpers spärlich, verästelt, anastomosierend; die Farbe ihres Pigments erscheint hell chromgelb; in manchen ist ein Kern deutlich. An der Vorderfläche des Schenkels sind die Zellen in großer Anzahl so gruppiert, daß sie den Ausführungsgang einer Hautdrüse einschließen. Sie haben hier die gewöhnliche grauweiße Farbe der Epithelzellen und in ihnen ist das or angefarbene Pigment an umschriebener Stelle angehäuft, während der Rest der Zelle grauwe i $B$ erscheint. Da an diesen Stellen keine Drüsenzellen vorkommen scllen und die Form der Zellen an jene erinnert, sollen nach $B$ i m m e rm a $n$ diese Pigmentzellen nichts anderes sein als „Hautdrüsenepithelzellen“.

E h rm a n n (1892, S. 523 f.) stellt Guanin und Lipochrom als die heilen Pigmente dem schwarzen gegenüber. Jene sollen in den polygonalen Zellen der Rückenhaut in e i $n$ e $m$ Zellkörper vereinigt sein. An den Uebergangsstellen der Rückenhaut in die weiße Bauchhaut und auf dieser selbst wird das Guanin und hier und da auch dás gelbe Pigment in eigenen, mehr verzweigten Zellen getrennt gefunden. Bei $R$ ana esculenta ist die Form der Zellen etwas weniger polygonal als bei $\mathrm{H}$ y $\mathrm{l}$ a. Das Mengenverhältnis der hellen Pigmente ist bei $\mathrm{Hyla}$ sehr konstant, indem in jeder Zelle das weiße und das gelbe Pigment immer im selben Verhältnis vorhanden sind, während es bei $R$ a n a escule n t a graue Individuen gibt, denen das gelbe Pigment größtenteils fehlt, und grüne, bei denen es reichlich vorhanden ist. E h rm a n n nimmt an, daß diese beiderlei Pigmente verschiedene Lagen in der Zelle einnehmen können. Im $\mathrm{gr}$ a u e $\mathrm{n}$ $Z$ ustande der Haut findet man das weiße und das gelbe $P$ i g m e $n t$ so innig durcheinander $g$ e $m$ i s $c h t$, daß man die Körnchen schwer voneinander unterscheiden kann, während in dem $\mathrm{g} \mathrm{e} / \mathrm{b} \mathrm{gr}$ ü $\mathrm{n}$ e $\mathrm{n}$ $Z$ ustande die $Z$ ellen an dem oberen $R$ ande einen intensiv gelben Saum oder Reflex zeigen (S. 529). Der gra u e Zustand beruht also darauf, daß das gelbe $P$ igment in die Tiefe tritt und mit dem weiBen sich mischt, der gr ü ne darauf, daß sich eine größere Menge des gelben $P$ igmentes wieder an der Oberfläche der Zellen sammelt.

B i e d e rm a n n (1892, S. 461 f.) beschreibt die Xantholeukophoren als rundliche oder undeutlich polygonale Zellen von gelber Farbe, die etwa nach Art eines Pflasterepithels ein Mosaik bilden, ohne dab jedoch die einzelnen Elemente sich unmittelbar berühren. Jede Zelle ist dicht erfüllt von rundlichen oder ovalen Körnchen, die in eigentümlicher Weise quer gestreift erscheinen, als ob sie aus einzelnen übereinander gelagerten Teilstücken aufgebaut wären. Eine deutlich kristallinische Struktur konnte B i e de rm a n n nicht wahrnehmen, obschon er bemerkt, das optische Verhalten weise darauf hin. Der Kern kennzeichnet sich als heller Fleck in der Mitte 
des Zellkörpers. Außer diesen Körnchen enthält jede I n t.erf e r e n zzelle $\left.{ }^{1}\right)$ - so bezeichnet Biedermann im AnschluB an Br ücke die Xantholeukophoren - noch ein goldgelbes Pigment in Form von größeren und kleineren Tropfen, welche je nach der Färbung der Haut sehr auffällige Lageveränderungen zeigen.

Bei vorherrschend $g r$ ü ne $r$ oder gelber Farbe bildet nach B i e derman in jeder Zelle die Schicht der I $n$ terfere $n z k$ örper

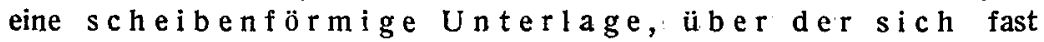
genau ihrer Ausdehnung entsprechend, das gelbe Pigment a usb r e it e t, so daß die Zelle gelb erscheint, ohne daß man die einzelnen diese Färbung verursachenden Tröpfchen deutlich sieht. Man kann sie aber leicht durch Auflösung der Guaninmassen in Kali- oder Natronlauge sichtbar machen. Die gelben Tröpfchen widerstehen der Einwirkung dieser

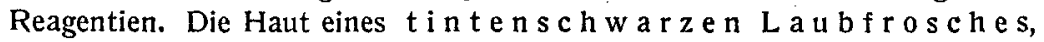
der durch Einwirkung höherer Temperatur sich $r$ asch a ufgehelit hat, unterscheidet sich von der Haut eines hellgelben Laubfrosches unter sonst gleichen Umständen vor allem durch

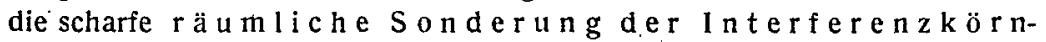
chen und des gelben Pigmentes, welch letztes nun nicht mehr diffus zerstreut über dem ersten liegt, sondern zu rundlichen Klumpen geballt, die Zwischenräume (!) zwischen den scheibenförmigen Massen der Interferenzkörner teilweise erfüllt. In jeder Zelle hat sich nämlich das gelbe Pigment zu einem Klumpen geballt, welchen man an den meisten Stellen von einer ungefärbten feinkörnigen Plasmamasse umschlossen und den Interferenzkörnern dicht angelagert findet, so daß es oft zweifelhaft erscheinen könnte, ob beides, die Interferenzkörnchen und das gelbe Pigment. wirklich nur Einschliisse einer Zelle sind, oder ob es sich nicht um zwei verschiedene, aber dicht nebeneinander liegende Zellen handelt. (!) Obwohl nun $B$ i ederma n n anführt, daß bei gewissen Fischen Interferenzkörnchen und gelbe Tröpfchen in völlig getrennten Zellen vorkommen, besteht nach ihm bei $\mathrm{Hyla}$ kein Zweifel, daß die Interferenzkörner und das

1) Hier möge eine historische Notiz über das Wort Interferenz$z$ e ll e n Platz finden. B r ü cke (1852) verstand unter diesem Namen $z$ we i e r le i ganz verschiedene Dinge: bei dem Chamäleon (S. 195-196) in der Epidermis gelegene I ufthaltige $Z$ ellen, die vermöge dieses Luftgehaltes zu Interferenzerscheinungen Veranlassung geben; diese Elemente sind die sog. Häutungszellen (vgl. W. J. S c h m i.d t, 1917, S. 108 Anmerkung). Von diesen Elementen sagt B r ï c k e (S. 196: "Diese Zellen ...... will ich schlechtweg Interferenzzellen nennen:" Von den Fröschen und insbesondere vom Laubfrosch berichtet $\mathrm{B} \mathrm{r}$ ii $\mathrm{ck}$ e etwas später (S. 196-197), daß hier unter der Epidermis aber über dem schwarzen Pigment Zellen liegen, deren feinkörniger $\mathrm{krist}$ a $1.1 \mathrm{i} \mathrm{n} \mathrm{i-}$ $s c_{h}$ e $r$ I n h a lt Interferenzerscheinungen bedinge (d. s. unsere Xantholeukophoren). $\mathrm{Da} \mathrm{B} \mathrm{r}$ ü $\mathrm{cke}$ auch hier von Interferenzzellen spricht, so ist die Bezeichnung in zweierlei, ganz verschiedener Bedetıtung gebraucht und daher am besten fallen zu lassen. 
gelbe Pigment Einschlüsse ein und derselben Plasmamasse darstellen. B i e derm a n n kommt zum Ergebnis, daß die gelbe und hellgraue Färbung beim Laubfrosch hauptsächlich durch eine $\mathrm{v}$ e r $\mathrm{s} c \mathrm{~h}$ i e d e n e rä u mliche Verteilung des in den Interferenzzellen enthaltenen g e l be n P i g m e $\mathrm{n}$ es bewirkt wird, indem dieses letzte sich bald über größere Flächen glëichmäßig verbreitet, bald nur örtlich an beschränkten Stellen anhäuft und dann für die Gesamtfärbung bedeutungslos wird, die in diesem letzten Falle wesentlich durch die Interferenzkörnchen bedingt wird. Aber auch die I n terfere $\mathrm{nzk}$ ör n chen erleiden hierbei gewisse $\mathrm{L}$ a gever änderungen: bei hellgelber Hautfarbe glitzern sie lebhaft in auffallendem Licht, während sie bei hellgrauen Fröschen ganz matt erscheinen; bei diesen drängen sich die Körnchen viel dichter zusammen, womit eine, wenn auch nicht sehr auffallende, V e r k l e i n e r u n $g$ der Zellen Hand in Hand geht.

G a u p p (1904, S. 500) unterscheidet auf Grund der vorliegenden Arbeiten (im Literaturverzeichnis erwähnt er auch die Untersuchung $F \mathrm{i}$ calbis!) Xanthophoren, die nur gelbes Lipochrom enthalten, L e u $\mathrm{k}$ o h o ren, die nur Guaninkörnchen umschließen, und $\mathrm{X}$ a $\mathrm{n}$ th $\mathrm{o}_{\text {- }}$ le uk ophoren, in denen das Lipochrom mit den Guaninkörnchen. in denselben Zellen vorkommt, wobei er sich in der Bezeichnung $\mathrm{Ke} 11 \mathrm{er}(1895, \mathrm{~S} .147 \mathrm{f}$.) anschließt und für die Interferenzzellen Biedermanns im Sinne der Kellerschen Xanthophoren und Leukophoren die Bezeichnung $X$ antholeukophoren einführt. Diese Xantholeukophoren sollen sich an den grünen Hautstellen finden. Des weiteren bringt dann $G$ a upp die Angaben $B$ i edermanns und Ehrmanns.

He m p e $1 \mathrm{~m}$ a n n $(1908$, S. 8) schließt sich in seinen Ausführungen über die Chromatophorenverhältnisse beim Frosch ganz an $G$ a u p p an und unterscheidet demnach Xanthophoren, Leukophoren und Xantholeukophoren, in welch letzten Guanin und Lipochrom nebeneinander vorkommen sollen.

Schließlich ist noch Siedle cki $(1909$, S. 710) als Vertreter dieser Auffassung zu nennen. Beim javanischen Flugfrosch (P ol y p ed a tes $r e i n w a r d i i$ ) findet er die von $B i e d e r m a n n$ beim Laubfrosch beschriebenen Verhältnisse und Vorgänge wieder. Die Xantholeukophoren liegen in einschichtiger, nur stellenweise zweischichtiger, Lage dicht unter der Epidermis. Etwa 6-8 von ihnen treten mit den Ausläufern einer Melariophore in Kontakt, die sich gewöhnlich unter der Xantholeukophorenschicht befinden; jedoch soll „das Umfließen der gelben Zellen durch die amöboiden Auṣläufer der Melanophoren" auf „präformierten Bahnen" erfolgen und beim Braunwerden der Tiere soweit gehen, daß der ganze Plasmaleib samt dem Kern auf die Xantholeukophore ,überwandert" und unmittélbar unter die Epitheloberfläche zu liegen kommt (? Sch.). Die Xantholeukophoren sind gewöhrilich halbkugelig, mit der flachen Seite dem Epithel angeschmiegt. Wo sie dichter liegen, werden sie durch gegenseitigen Druck in mehr prismatische Gebilde umgewandelt; jedoch bleibt der untere, dem Korium zugewandte Teil derselben immer halbkugelig. Die $\mathrm{Z}$ elle $\mathrm{n}$ e $\mathrm{n} \mathrm{th}$ a 1 t e $\mathrm{n}$

Archiv f. mikr. Anat. Bd. 93. Abt. I. 
in regelmäßigen parallelen Schichten die $G$ u aninkörnchen und a uBerdem das gelbe Lipochrom zwischen diesen Schichten (vorwiegend im unteren Teil der Zellen) in der Form vom Tröpfchen. Dicht an der Oberfläche der Xantholeukophoren liegt der linsenförmige Kern.

So verhalten sich nach $\mathrm{S}$ i e d l e c k i die Dinge an den dunklen Hautstellen; an den hellgefärbten dagegen sind die Xantholeukophoren in ellipsoidale Gebilde umgewandelt, deren Kern als kompakter Körper tief unten im Plasma liegt. Zwischen beiden Zuständen finden sich Uebergänge. Daraus schließt $\mathrm{S}$ i e d l e cki, dab die Xantholeukophoren ihre Gestalt veränder $n$ können und dabei der Kern von ihrer Oberfläche in die Tiefe des Plasmas wandert. Bei dieser Wanderung des Kernes werden die parallelen Lamelien stark umgebogen, so daß sie in einer Zelle, deren Kern sich schon ganz unten befindet, einige Anhäufungen bilden, an denen nur noch Spuren der konzentrischen Schichtung sichtbar sind. Die glitzernden Guaninkörnchen befinden sich in den ausgebreiteten Zellen vornehmlich in der nächsten Umgebung des Kernes, wogegen die tieferen Schichten von dem Lipochromtröpfchen eingenommen sind. Die Guaninkörnchen geben den Zellen einen bläulichen Schimmer, der noch dadurch verstärkt wird, daß sich der stark lichtbrechende Kern über demselben befindet. Der gelbe Farbstoff ist vorwiegend unter den Körnchen ausgebreitet; bei dieser Lage muß also die blaue Färbung überwiegen und eine intensive dunkelbläulichgrüne Hautfarbe daraus resultieren. Sobald jedoch die Kerne der Xantholeukophoren in die Tiefe der Zellen gewandert sind, werden die Guaninkörnchen infolge der Verschiebung des Plasmas von den gelbes Pigment führenden Schichten überdeckt; auf diese Weise entsteht die lichte gelbgrüne Hautfarbe.

Diesen sehr bestimmt gehaltenen Aeußerungen $\mathrm{S}$ i c d l e c k is gegenüber muß ich eine gewisse Zurückhaltung beobachten, weil sie einen Frosclis betreffen, der unseren heimischen Formen schon ferner steht. S i e d l e c $k$ i s Schnittbilder geben eigentlich nur über das Verhalten des Guanins Aufschluß. Sollten seine Angaben über das Nebeneinander von Lipochrom und Guanin in derselben Zelle auf Beobachtungen am lebenden Objekt beruhen. so dürfte auch hier unbedingt eine Nachprüfung notwendig werden, nachdem sich herausgestellt hat, daß bei unseren einheimischen, so viel untersuchten Fröschen gewandte Beobachter bei der Deutung der Xantholeukophoren sich getäuscht haben.

Fuch s (1914, S. 1483) stellt zusammenfassend die Verhäitnisse so dar, daß ein Teil der älteren Forscher den komplizierten Bau der Xantholeukophoren nicht immer richtig erkannt habe, in dem sie diese Zellen- in zweierlei Elemente unterschieden, was neuerdings $\mathrm{Fic}$ al bi wieder im Gegensatz zu Ehrmann und $B$ i edermann annehme. Da $F u c h s$ Fic a l b is Arbeit nicht im Original zugängig war, begnügt er sich mit einem einfachen Hinweis auf dessen Angabe und betont, daß das Verdienst, den richtigen Sachverhalt erkannt zu haben, $\mathrm{Hering}$ gebührt. Fuch s vertritt also durchaus den Standpunkt $B$ i edermanns und Ehr$\mathrm{manns}$. - Die Abbildungen von Ehrmann und Biedermann sind in dic zusammenfassende Literatur übergegangen $(G$ a u p p, H e m- 
p e I m an n, Fuch s) und haben die irrige Auffassung von der Existenz der Xantholeukophoren befestigt.

Für eine Verteilung von Guanin und Lipochrom auf $z$ weierle i Zellen sind v. Wittich, vielleicht auch Winkler und $\mathrm{Pouchet}$ vor allem aber Fic a $1 \mathrm{bi}$ und im Anschluß an den letzten $v$ an $R$ ynberk in seiner zusammenfassenden Darstellung eingetreten.

v. W it t i ch (1854 a, S. 42 u. f.) hat zweifellos die guaninhaltigen und lipochromhaltigen Zellen auch an den grünen Hautstellen auseinander halten können. Er unterscheidet beide als g e l b e Pigmentzelle $\mathrm{n}$ und als In terferenzzelle $n$, gibt aber (im Gegensatz zu Hering und anderen, s. o.!) an, daß b e i d e sehr schnell sowohl durch Säuren als durch Alkalien zerstört werden. Doch neigt er zur Annahme, daß beide Zellarten nur' Entwicklungszustände ein und derselben Zellform seien, einmal auf Grund des erwähnten mikrochemischen Verhaltens, dann aber auch deshalb, weil ïberall da, wo gelbe Zellen auftreten, unter Umständen statt derselben Interferenzzellen vorkommen können. Diese Meinung vertritt $\mathrm{v}$. Wit $\mathrm{t} i \mathrm{ch}$ auch in einer späteren Arbeit (1854 b, S. 259), indem er sagt: ,Wie ich schon früher angab, scheinen diese Interferenzzellen in einem gewissen genetischen Zusammenhange mit den gelben Fettzellen zu stehen, nicht allein, daß wir unter den Erscheinungen einer Art Atrophie die gelben Zellen fast ganz verschwinden sehen urid an ihrer Stelle die sehr geschrumpften Interferenzzellchen finden, bekommt man auch sehr oft unter ganz normalen Verhältnissen gelbgefüllte Zellen zur Beobachtung, die noch nebenher kristallinische Flitterchen enthalten und es diesen verdanken, daß sie bei durchfallendem Lichte teilweis undurchsichtig, bei auffallendem teils gelb, teils auf weißem Grunde schillernd erscheinen." Nach solchen Aeußerungen zu schließen, läßt v. Witt i $\mathrm{ch}$ aūch das gleichzeitige Vorkommen von Guanin und Lipuchrom in einer Zelle zu und kann demnach nur mit Einschränkung als ein Vertreter der, zweiten Richtung betrachtet werden.

P ouchet $(1876$, S. 55 f.) weist in der grünen Haut der Frösche Lipochrom (pigment jaune) und Guanin offenbar verschiedenen Zellen zu. Das ölärtige, gelbe, in Alkohol lösliche Pigment bilde Tropfen in den Chromatophoren, die bei der Präparation sehr groß werden. Doch sei es schwer, die Zellen, welche dieses gelbe Pigment enthalten, einzeln zu beobachten, wenigstens beim erwachsenen Tier.

W i n k l e r (1910 a, S. 256), der hier zunächst genannt werden soll, dem aber $F$ icalbis Arbeit bekannt ist, entscheidet sich nicht klar für diesen Autor oder für B i edermann, sondern empfiehlt, „aus praktischen Gründen" nicht drei Schichten von Pigmentzellen (Xanthophoren, Leukophoren, Melanophoren), wie Ficalbi will, sondern nur zwei, Xantholeukophoren, mit Guanin und Lipochrom erfüllt, und Melanophoren zu unterscheiden. Auch an einer anderen Stelle $(1910 \mathrm{~b}$, S. 626) zeigt Winkler die gleiche Unentschiedenheit: er führt in der Rückenhaut $z$ wei oberflächliche Schichten, Xanthophoren und 
Leukophoren, und darunter eine tief liegende Schicht von Melanophoren an. Doch spricht er wenige Zeilen danach wieder von Xantholeukophoren (S. 627). Aus den Abbildungen läßt sich keine Klarheit über die Stellungnahme des Autors zu unserer Streitfrage gewinnen.

van Ry n berk (1906, S. 493) ist der einzige Autor, welcher die nun anzuführenden Angaben $\mathrm{Fic}$ a I b is (1896) richtig gewürdigt hat, sehr wahrscheinlich deshalb, weil ihm (im Gegensatz zu F u c h s) die Arbeit im Original vorlag und er wohl auf Grund der Abbildungen zur Ueberzeugung kommen mußte, daß diese Beschreibung ,eine sehr wertvolle Berichtigung der $\mathrm{B}$ i e derman $\mathrm{n}$ schen Untersuchung" bilde. $v$ a n $R$ y n berk gibt den im folgenden wiederholten, anscheinend wörtlich übersetzten Auszug aus $F$ i c a l b.is Monographie. Fic a l bi unterscheidet in der oberen Schicht der Kutis nicht $z$ w e i Lagen von Pigmentzellen, wie B i edermann, sondern drei: „Die erste oder oberflächliche Schicht besteht aus sphärischen Chromatophoren mit deutlichem Kern. Sie sind sehr nahe aneinander gelagert, aber berühren sich nicht, so daß enge reelle oder virtuelle Spalten zwischen ihnen übrig bleiben. Auf einem Querschnitte erscheinen sie in der Mitte am dicksten; bei der Flächenansicht zeigen sie aber eine vieleckige Zellform, einem platten Epithel ähnlich. Diese Chromatophoren sind gelb, meist goldgelb oder zitronengelb, und der Farbstoff ist in zahlreichen feinen Tropfen enthalten. . . . . . . . Die zweite oder mittlere Schicht besteht ebenso aus sphärischen Chromatophoren mit deutlichem Kern. Ihre Größe ist jener der über ihnen gelagerten gleich und sie erscheinen in den.Querschnitten halbmondförmig, weil ihre obere Fläche nach oben konkav ist und ein Bett bildet, worin je eine Chromatophore der oberen Schicht gelagert ist. Sie sind von violetter Farbe, wie der Bodensatz des Weines, sind aber irideszierend und können andere Farben zeigen. Ihr Farbstoff ist in großen Körnchen enthalten, welche untereinander in den Pigmentzellen zu einer. Art Farbenspiel Veranlassung geben. . . . . . Die dritte Schicht ist jene der schwarzen . . . . . . Chromatophoren. Diese haben einen kernhaltigen Körper (der Kern ist oft schwer zu sehen) und verzweigte Fortsätze. Sie bilden eine sparsame Schicht, da die Körper der schwarzen Pigmentzellen einander nicht berühren. Die Fortsätze tun es aber und daher sieht man auf den Querschnitten eine kontinuierliche, unter die beiden beschriebenen Chromatophorenschichten gelagerte schwarze Schicht. An Flächenpräparaten sieht man aber, daß die Schicht in der Tat sparsam ist, so daß nur je eine schwarze Chromatophore mehreren darüber gelagerten entspricht."

Leider konnte auch ich $\mathrm{Ficalbis}$ Arbeit (erschienen in Atti della R. Accademia Peloritana in Messina, 1896) nicht im Original einsehen, sondern meine Kenntnis derselben erstreckt sich auf das, was van Ry n berk darüber sagt. Das vorstehende Zitat ergibt aber im Vergleich mit meinen eigenen Befunden unzweifelhaft, daß Fic albi die eigentümliche Form und Lagebeziehung der zu einer scheinbaren Xantholeukophore vereinigten 
Xanthophore und Guanophore richtig. erkannt hat ${ }^{1}$ ). Diese Feststellung ist aber, in der deutschen Literatur wenigstens, nicht zur Anerkennung gelangt, obwohl die Arbeit $F$ i c al bis schon mehr als 20 Jahre zurückliegt, eben weil sie an so schwer zugänglicher Stelle erschien. Da meine Befunde ganz unabhängig von denen Fic a 1 b is gemacht wurden - ich lernte die bei van $\mathrm{Ryn} b$ erk zitierte Stelle aus $\mathrm{Ficalbi}$ erst genauer kennen, nachdem ich meine Beobachtungen abgeschlossen hatte und mich in die literarische Seite der Frage vertiefte - und da sie ferner in mancher Hinsicht eine Ergänzung der $\mathrm{Fic}$ a $1 \mathrm{~b}$ i schen darstellen dürften, so wird die folgende Mitteilung wohl willkommen sein.

\section{Eigene Untersuchungen.}

Schon seit Jahren hatte ich Zweifel an der Richtigkeit der Ehrmann-Biedermannschen Auffassung - als solche will ich die eingangs erwähnten Anschauungen kurz bezeiclinen - und zwar aus folgenden Gründen. Bei den Reptili e n kommt die gr ü ne Farbe in folgender Weise zustande; eine Lage lipo= chromführender Zellen (Lipophoren $=$ Xanthophoren) ist über einer Lage guaninhaltiger Zellen (Guanophoren = Leukophoren) ausgebreitet; darunter folgt noch eine Schicht von Melanophoren, die einen schwarzen Hintergrund liefert, vor dem die Guanophoren in auffallendem Licht $b \mathrm{l}$ a $u$ erscheinen. Diese blaue Farbe wird durch die Ueberdeckung mit Gelb in Grün verwandelt. Wäre also die Ehrmann-Biedermann sche Auffassung richtig, dann läge das eigentümliche Verhalten vor, daß der auf der Ueberlagerung zweier bestimmter Substanzen (Guanin und Lipochrom) beruhende färberische Effekt in dem einen Falle (Amphibien) durch schichtweises Ueberlagern der beiden Stoffe in e i ne $r$ Zelle, im anderen Falle (Reptilien) durch Ueberschichtung der gleichen farberzeugenden Stoffe in zwei übereinandergelegenen Zellschichten hervorgerufen würde.

Ferner ist $z u$ bedenken, daß Guanin und Lipochrom chemisch

1) Hinsichtlich der Deutung des Inhaltes der Guanophoren (Leukophoren) scheint $\mathrm{Fic}$ albi weniger das Richtige getroffen zu haben. Möchte man doch vermuten, daß er hier an ein richtiges Pigment denkt, da er von „Farbstoff" spricht (s. obiges Zitat); andererseits hebt er allerdings auch das Irisieren dieser Zeilen hervor. 
sehr verschiedene Körper sind; daß sie nebeneinander in derselben Zelle gebildet werden, ist zwar von vornherein nicht unmöglich, aber insofern unwahrscheinlich, als bei Fischen, worauf ja schon Bi e d e rmann (s. o.) hingewiesen, und Reptilien durchweg Guanin und Lipochrom auf zweierlei typisch verschiedene Zellen verteilt sind ${ }^{1}$ ). Dazu kommt noch, daß auch beim Laubfrosch neben den Xantholeukophoren Xanthophoren - einzig mit Lipochrom und Leukophoren - nur mit Guanin allein - vorkommen (s. o. Ehrmann). Soll unter diesen Umständen die Vermutung nicht nahe liegen, daB F i c a I b i doch recht hat, und die Xantholeukophoren eine Kombination von Xanthophoren und Leukophoren darstellen?

$\mathrm{Zu}$ diesen durch morphologische Gründe erweckten Zweifeln kommt noch die physiologische Schwierigkeit sich vorzustellen, daB die Guanin- und Lipochrommassen in den Xantholeukophoren sich bei den intrazellulären Verlagerungen des Lipochroms (s. 0. Bi ederma $\mathrm{n}$ ) nicht regellos miteinander vermischen, oder wenn eine solche Vermengung eingetreten ist (was nach $\mathrm{Ehrmann}$ und $\mathrm{Si}$ e d le c k i vorkommen soll s. o.), sich wieder reinlich $z u$ scheiden vermögen. Alle diese Schwierigkeiten fallen hinweg, wenn Guanin und Lipachrom sich in verschiedenen Zellen befinden.

So ging ich denn mit der festen Erwartung an die Untersuchung heran, daß die Xantholeukophoren des Laubfrosches keine einheitliche Zellform darstellen, sondern durch Ueberlagerung von Xanthophoren und Leukophoren vorgetäuscht werden. Diese $\mathrm{Er}$ wartung bestätigte sich vollkommen.

Als Untersuchungsobjekt diente mir ein männlicher Laubfrosch, der beim Töten eins schöne $\mathrm{g} r$ ü $n$ e Farbe hatte. Alle folgenden Angaben beziehen sich demnach auf den $\mathrm{g} r$ ü n e $\mathrm{n}$ Zustand der Haut. Ich bemerke ausdrücklich, daß gewisse andere Farbtöne (silbergrau) sich hinsichtlich der A nordnurg von Guanin und Lipochrom in der Haut wesentlich vom grünen Zustand unterscheiden; doch bleiben unter allen Umständen - und das ist hier der wesentliche Punkt - Guanin und Lipochrom auf verschiedene Zellen verteilt.

1) Nur W a g ne r (1911, S. 28) berichtet, er habe Guanin und Lipochrom bei (sehr jungen) Forellen in derselben Zelle nebeneinander beobachtet; allerdings läßt er hinsichtlich der Guaninnatur der beobachteten Körnchen Zweifel offen. 
Zunächst prüfte ich grüne $\mathrm{H}$ a u t $\mathrm{s} t$ ü $\mathrm{c} \mathrm{ke}$, vornehmlich von der Dorsalseite des Oberschenkels, die dem soeben getöteten Tier entnommen waren, möglichst glatt, mit wenig Wasser und die Epidermis nach oben auf dem Objektträger ausgebreitet, unter starken Vergrößerungen in $\mathrm{F} l$ ä $\mathrm{c}$ h e $\mathrm{n}$ c $\mathrm{n} \mathrm{s}$ i $\mathrm{ch} \mathrm{t}$. Man kann hierbei sehr gut Immersionen benutzen, wenn man für hinreichend starke Beleuchtung Sorge trägt. Bei Anwendung einer Liliputbogenlampe von Leitz, die mit Gleichstrom von 3-4 Ampere gespeist wurde, ergab der $Z$ e i $B$ sche Apochromat $2 \mathrm{~mm} \mathrm{~N}$. A. 1.30 mit Kompensationsokular 4 oder 8 ausgezeichnete Bilder. Ein Entfernen der Epidermis oder. Anwendung. aufhellender Flüssigkeiten (Glyzerin) ist vollkommen überflüssig.

Die Xantholeukophoren erscheinen in solchen Präparaten an grünen Hautstellen als rundlich-polygonal umgrenzte.Gebildə, die bei der starken Beleuchtung (unter schwächeren Vergrößerungen) in prächtigen Interferenzfarben erstrahlen. Untersucht man sie mit starken Objektiven, so lassen sich deutlich 2 horizontal übereinandergeschichtete, verschiedenartige Anteile dieser Elemente unterscheiden: bei hoher Einstellung, also bei Untersuchung der der Epidermis zugewandten Seite, gewahrt man sehr dichtliegende, selbst bei tausendfacher Vergrößerung immer noch klein erscheinende, gelbe Körnchen, das Lipochrom; beim Senken des Tubus gelangt man dagegen in ein Gebiet, das mit viel gröberen körnigen Massen erfüllt ist, die aus Guanin bestehen. Während sich in den Guaninmassen fast immer eine helle Stelle auffinden läßt, die dem Ort des Kernes entspricht, sucht man gewöhnlich im Niveau der gelben Körnchen, des Lipochroms, vergeblich nach der Andeutung eines Kernes.

Sehr eigentümlich und meiner Erwartung zunächst entgegen war die Tatsache, daß die Areale der zu einer „Xantholeukophore“ gehörigen Guanin- und Lipochrommassen sich in ihrem Umfang genau decken, was ja auch Biederma n n hervorhebt (s. o.). Wenn es sich um die Ueberlagerung einer Guanophorenschicht durch eine Lipophorenschicht handeln sollte, wie ich annahm, war es sehr erstaunlich, daß doch zwischen den Lipophoren und den Guanophoren derartig enge räumliche Beziehungen bestehen, wie sie sich aus der genauen Einhaltung des gleichen Areals übereinandergelegener Zellen unzweifelhaft $z u$ erkennen gaben. So einfach wie bei den Reptilien, bei denen der letzt erwähnte Umstand nicht festzustellen 
ist, konnten demnach die Verhältnisse hier nicht liegen. Andererseits beobachtete ich aber im Uebergangsgebiet von grünen $z u$ gelben Hautstellen ( = vom Rücken zu den Körperseiten), daß allmählich diese enge. Beziehungen zwischen. Lipochrom- und Guaninverteilung in den „Xantoleukophoren“ verloren gehen: man sieht nämlich einzelne Elemente, in denen'die Masse der gelben Lipochromkörnchen sich nach Art von Ausläufern über das Gebiet der zugehörigen Guaninmasse hinaus erstreckt und solche Zustände führen schrittweise über $\mathrm{zu}$ einer vollständigen Trennung von Guanophoren und Lipophoren, wie sie schon $\mathrm{E}$ h $\mathrm{r} \mathrm{m}$ a n $\mathrm{n}$ (s. 0.) bekannt war.

Man kann die bisher geschilderten Verhältnisse auch an Hautstücken beabachten, die kurz mit absolutem Alkohol und Xylol behandelt, dann in Balsam übergeführt werden. Der Lipochromfarbstoff bleibt in solchen Totalpräparaten wenigstens eine kurze Zeit erhalten.

An zweiter Stelle untersuchte ich $20 \mu$ dicke Ko h le n s ä u r eGefrierschnitte von der grünen Haut der Dorsalseite des Oberschenkels, die 12 Stunden in $10 \%$ Formol fixiert worden war. Formol erhält die gelbe Farbe, wenn der Farbstoff auch nicht unverändert bleibt, inscfern als die Körnchen viel größer sind, als sie im überlebenden Objekte und im eben erwälınten, kurz mi† Alkohol fixierten Totalpräparat erscheinen; man gewinnt den Eindruck, daß die kleinen Lipochromtröpfchen zu größeren zusammengeflossen sind. Um gute Schnitte durch die dünne Haut zu erhalten, bildete ich aus einem Hautstückchen eine Rolle und schnitt diese senkrecht zu ihrer Längsachse. Die Schnitte rollten sich, in Wasser untersucht, wieder ab und zeigten sehr schön in jeder "Xantholeukophore“" die beiderlei Substanzen horizontal übereinander geschichtet, das gelbe Lipochrom in Form eines Streifens an der Epidermisseite, darunter die kristallinischen Guaninmassen (Fig. 1, Taf. IV). Die Grenze der beiden Stoffe ist absolut scharf, allerdings nicht ganz geradlinig. Niemals konnte ich eine Vermengung beider Substanzen, wie sie doch zu erwarten wäre, wenn sie in ein und derselben Zelle beieinander lägen, und auch von $\mathrm{Eh} \mathrm{rm}$ a $\mathrm{n}$ gefordert wird (s. o.), beobachten. Ferner traten jetzt, nach der Formolfixierung, an vielen Stellen i n e $\mathrm{rha} / \mathrm{b}$ der gel b e Li p o chrommassen unzweifelhaft kenntlich, $Z$ el $1 \mathrm{kerne}$ hervor. Die Stellen der Kerne in den Guaninmassen, die am Flächenpräparat der Haut so leicht festzustellen waren, konnte ich hier 
nicht regelmäßig beobachten, dafür waren die Schnitte zu dick; jedoch gewahrte ich an einzelnen Stellen, daß innerhalb e i n e $r$ "Xantholeukophore" sowohl im gelben Pigment als auch im Guanin je ein Kern lag, somit auf jedes Element $z$ wei Kerpe entfallen. Diese Tatsache zusammengehalten mit der guten $\mathrm{Ab}$ grenzung der beiderlei Substanzen zeigt wohl schon überzeugend, daß jede ,Xanthole ukophore“ eine Kombination $z$ weier Zellen, einer Xanthophore und einer Le u k o p hore, darstellt.

Im Gefrierschnitt waren die engen Beziehungen zwischen einer Guanophore und der darüber gelegenen Xanthophore, die sich in der Einhaltung des gleichen Arenals in der Flächenansicht ausprägten (s. o.), noch besser zu erkennen (Fig. 1, Taf. IV). Jede Lipophore sitzt ihrer Guanophore wie eine Kappe auf. Die Lipophore (X) springt etwas konvex gegen die Epidermis und gegen die Guanophore vor, so daß ihre Gestalt etwa als linsenförmig bezeichnet werden kann. Die Guanophore (L) ist auf ihrer Oberseite entsprechend ausgehöhlt, auf ihrer Unterseite gerundet und gegen die Melanophoren (M) vorgedrängt. Diese innige Beziehung, welche offenbar zwischen je einer Leukophore und einer Xanthophore besteht, läßt sich einigermaßen den Kombinationen von verschiedenartigen Farbzellen vergleichen, wie sie von B allow it z (1913 a und c) bei Fischen beschrieben worden sind. Wenn diese räumliche Beziehung der beiderlei $z$ u einer "Xantholeukophore" vereinigten Zellen auch nicht vollkommen erklärt werden kann, so wird sie doch verständlicher durch die.Tatsache, daß zwischen den "Xantholeukophoren" die sogenannten aufsteigenden Fasern der Lederhaut zur Epidermis emporstreben und somit gewissermaßen kleine Fächer gebildet werden; die in gleicher Weise den Xanthophoren und den Leukophoren zur Ausfüllung zur Verfügung stehen.

Ich versuchte auch, derartige Gefrierschnitte, ungefärbt oder leicht mit Thionin gefärbt, in Balsam $z \mathbf{u}$ überführen; doch war trotz beschleunigter Behandlung das gelbe Pigment stets verschwunden. Dieser Unterschied gegenüber dem Totalpräparat (s. o.) ist wohl so zu erklären, daß an den Schnitten Alkohol und Xylol viel leichter Zutritt zum Pigment haben, als an ganzen Hautstücken, und der Farbstoff mit ständig wechselnden Mengen des Lösungsmittels in Berührung kommt, ferner auch wohl durch die Formol- 
behandlung und das Gefrieren, wodurch eine Veränderung (Verklumpung) der Lipochromgranula hervorgerufen wird.

SchlieBlich stellte ich Paraffinschnitte her, zum Teil von formolfixierten Objekten, zum Teil von Hautstücken, die 12 Stunden lang mit einmal gewechseltem starken Fle $\mathrm{m} \mathrm{m}$ i n gschen Gemisch behandelt waren. Die letzte Fixierung erwies sich für die feinere Erhaltung der Lipophoren und Guanophoren vorteilhafter als die Formolbehandlung; doch konnten auch boi dieser die wesentlichen Verhältnisse durchaus deutlich erkannt werden. Zum Färben gebrauchte ich in beiden Fällen Thionin-Eosin, D e l af i e lds Hämatoxylin-Eosin oder van $G$ i e s o ns Gemisch, Polychromes Methylenblau nach Unna, Pappenheims Methylgrün-Pyronin, schließlich Eisenhämatoxylin nach $\mathrm{H}$ e i d e $\mathrm{n} h \mathrm{~h} i \mathrm{n}$, sei es allein oder in Verbindung mit Eosin oder $v$ a $n$ Gi e s o n s Gemisch. Am vorteilhaftesten von diesen Färbungen waren ThioninEosin und die Eisenhämatoxylinmethode mit den genannten Kombinationen; die letzte hat allerdings den Nachteil, daß die Guaninmassen durch längere Behandlung mit der Eisensalzbeize mehr oder minder aufgelöst werden. Für deren Untersuchung eignen sich daher mehr dje vorgenannten Färbungen, vor allem ThioninEosin, D e 1 a f i e l d s Hämatoxylin in Verbindung mit Eosin oder van Giesons Gemisch; auch $\mathrm{Pappenheimsche} \mathrm{Fär-}$ bung gibt hübsche Präparate unter Erhaltung des Guanins. Die Schnittdicke betrug durchweg $10 \mu$. Sowohl Quer- als Flachschnitte der Haut kamen zur Untersuchung.

Beginnen wir mit der Betrachtung eines mit Thionin und Eosin gefärbten $Q$ uerschnittes durch die Rückenhaut (Fig. 2, Taf. IV). Dicht unter der Epidermis (E), nur durch die dünne kollagene Grenzlamelle von ihr getrennt, liegt in sehr regelmäßiger Anordnung eine einfache Schicht von D o p p e lze lle n, von "Xantholeukophoren". Durch ihre Größe, Form und Färbung heben sie sich auffallend von allen anderen Elementen der Kutis ab. Jede Doppelzelle setzt sich zusammen aus einer zart bläulichgrün gefärbten $\mathrm{X}$ a n th o phore $(=$ Lipophore, $\mathrm{X}$, Fig. 2, Taf. IV) und einer gelblichen Le u k o p hore (= Guanophore, L). Jede $X$ an tho phore (X, Fig. 2, Taf. IV) hat die Form einer dicken bikonvexen Linse, deren obere Fläche gegen die Epidermis vorspringt, während die untere, stärker gewölbte, von der Guanophore (L) umfaßt wird. Die Lipophoren stehen dicht aneinander und 
sind nur durch feine Bindegewebssepten getrennt, welche von der kollagenen Grenzlamelle abgehen. Hin und wieder findet sich in diesen Septen der kleine Kern einer Bindegewebszelle (vgl. Fig. 2, Taf. IV, zwischen den beiden mittleren Doppelzellen). Durch dieses dichte Aneinanderlagern der Zellen kommt vielfach der Rand der Linse in Wegfall, und, wie die Flachschnitte lehren (s. u.), bedingt dieses enge Aneinanderschließen der Xanthophoren ihre gegenseitige polygonale Begrenzung.

Der zweite Bestandteil der Doppelzelle, die L e u o phore (L, Fig. 2, Taf. IV), erscheint im Schnitt halbmondförmig und schmiegt sich der unteren stärker gewölbten Fläche der Xanthophore an. Räumlich betrachtet ist die Leukophore becherförmig; die Höhle dieses Bechers wird von der Xanthophore ausgefüllt.

Die Xanthophoren und die Leukophoren einer jeden Doppelzelle sind $\mathrm{nicht}$ durch Bindegewebslamellen voneinander geschieden, während solche feinen Häutchen benachbarte Doppelzellen voneinander trennen.

Faßt man die ganzen Doppelzellen (an grünen Hautstellen) ins Auge, so erscheint der Anteil der beiderlei Farbzellen daran im Querschnitt der Haut etwas verschieden. Gewöhnlich ist die Xanthophore voluminöser, die Leukophore weniger umfangreich. Dieses Verhalten gilt vor allem für die Rückenhaut. In der Haut von der Dorsalseite des Oberschenkels sieht man vielfach das Gegenteil davon; aber auch in der Rückenhaut finden sich gelegentlich ähnliche Vorkommnisse.

Unter den Guanophoren folgen die $M$ ela nophoren $(M$, Fig. 2, Taf. IV), die mit ihren Ausläutern guirlandenartig die Unterseite jener einfassen. In meinen Präparaten ist ihr Pigment ziemlich stark geballt und ihre Ausläufer lassen sich, pigmenterfüllt, nur bis zum Becherrand der Guanophore verfolgen, indem sie sich in die Lücken hineinschieben, welche zwischen den Halbmonden frei bleiben. Da die Doppelzellen bei dem geschilderten Zustand der Melanophoren in ihrem oberen Teil dicht aneinander stoßen, muß ihre Form eine andere sein, wenn die Ausläufer der schwarzen Pigmentzellen vollständig mit Pigment erfüllt sind: sie werden alsdann von den Seiten her durch das gegen die Epidermis vorflutende Pigment zusammengedrückt und müssen somit in der Richtung senkrecht zur Hautfläche an Ausdehnung zunehmen. Solche Formveränderung hat ja auch Bie derm a n n (s. o.) aus dem Studium der 
Flächenansicht erschlossen; in der Tat ist das Aussehen der Xantholeukophoren bei gewissen Färbungszuständen der Haut wesentlich von dem hier geschilderten verschieden.

Ganz ähnlich wie bei Thionin-Eosin-Färbung bietet sich das Bild eines mit Eisenhämatoxylin gefärbten Querschnittes dar (Fig. 3, Taf. IV), nur daß die Leukophoren weniger auffallen, weil ihr Inhalt, das in durchfallendem Licht im Schnitt gelbliche Guanin, sich gar nicht mehr oder nur schwach erkennen läßt. Ein gewisser Unterschied der beiden Schnitte (Fig. 2 u. 3, Taf. IV) liegt darin, daß bei dem letzten die Becher der Leukophoren im Allgemeinen näher ans Epithel heranreichen, bisweilcn die kollagene Grenzlamelle berühren. Die Ausläufer der Melanophoren treten aber auch hier nicht bis an die Epidermis heran.

Gehon wir nun auf den B a d e r e inzelnen Kom pone n'ten der Doppelzellen näher ein. Die $X$ anthophoren sind bei Fixierung mit Flemmings Gemisch und Färbung nach Pap pen hei m, oder mit Thionin-Eosin zart grünlichblau gefärbt, bei Tinktion mit Dela fi elds Hämatoxylin rötlichblau, bei Eisenhämatoxylinbehandlung merklich dunkler als die Leukophoren. Sie besitzen einen bläschenförmigen $\mathrm{K}$ e $\mathrm{r}$ n mit ein bis zwei Nukleolen und spärlichen kleinen Chromatinbröckchen. Der Kern ist gewöhnlich parallel zur Ebene der Haut abgeflacht und liegt in der Mitte der Zelle (Fig. 5 u. 7, Taf. IV). Gar nicht so selten findét man statt e i $n$ es Kernes $z$ we i nahe beieinandergelegene (Fig. 4 und 8, auch zwei Zellen in Fig. 3, Taf. IV).

Das Plasma der Xanthophoren erscheint bei ThioninEosin-Färbung fast homogen (Fig. 4 u. 5, Taf. IV), bei Eisenhämatoxylinbehandlung dagegen vor allen in der Umgebung des Kernes mehr oder minder deutlich gekörnt Fig. 7-9, Taf. IV). Oefter (Fig. 10, Taf. IV) war das Plasma gleichmäßig mit kleinen dicht gelegenen deutlich erkennbaren Gran u la erfüllt. Das konnte ich bei Eisenhämatoxylinfärbung nur ziemlich selten aber dann ganz klar beobachten, während bei Färbung mit $D$ e I a f i e $1 \mathrm{~d}$ s Hämatoxylin dieses Verhalten allgemein aber weniger deutlich kenntlich war. Da die gelbe Färbung aus den Schnitten stets verschwunden ist, kann es sich nicht um die in Alkohol löslichen Lipochromgranula handeln, sondern es liegt eine davon abweichende Körnung vor, die bei der Beobachtung des überlebenden Objektes meist nicht festzustellen ist. Es ist vielleicht nicht überflüssig, $z u$ betonen, daß die Körnchen 
kein Guanin sind. Ob sie vielleicht Vorstufen der gelben Körnchen darstellen, müssen weitere Untersuchungen lehren. Es sind aber zweifellos die Körnchen, welche B i e d e rm a n n (s. o.) als u $\mathrm{n}$ g e$\mathrm{f}$ är b t e, feinkörnige Plasmamasse bezeichnet und auch in seinen Abbildungen (Taf. XI, Fig. 2) angedeutet hat.

$\mathrm{DaB}$ den Xanthophoren $\mathrm{Z}$ entriolen zukommen, kann ich nicht mit Bestimmtheit behaupten. Oefter sah ich in der Nähe des Kernes einzelne kleine Körnchen oder auch ein Doppelkörnchen (Diplosom), das sich mit Eisenhämatoxylin stärker färbte als die anderen körnigen Einlagerungen des Plasmas. Doch habe ich dies Gebilde nicht mit der Regermäßigkeit gefunden, daß ich seine Natur als Zentriol über allen Zweifel sicher stellen könnte.

Noch ein paar Worte über die $Z$ weikernigkeit der Xanthophoren. Ob diese zwei Kerne auf mitotischem oder amitotischen Wege aus dem ursprünglich in Einzahl vorhandenen Kerne hervorgehen, läßt sich aus den morphologischen Verhältnissen nicht entnehmen. Ist aber ein Analogieschluß auf die zweikernigen $M$ eI a n o phoren bei Urodelen und Reptilien erlaubt, so liegt die Wahrscheinlichkeit mitotischer Entstehung vor. (Vgl. Pernitz s ch 1913, S. 173, S ch midt 1917, S. 139.) Zweikernige Xanthophoren sind bis jetzt noch selten beobachtet. Bei Reptilien (Lacerta) fand ich sie immer einkernig ( $\mathrm{S} \mathrm{ch} \mathrm{mi} \mathrm{d} \mathrm{t} \mathrm{1917,} \mathrm{S.} \mathrm{182).}$ Doch beschreibt B a 110 wit $z$ (1913 c, S. 546), daß bei Knochenfischen (Gobiiden) die Xanthophoren wie die Melanophoren gewöhnlich zweikernig sind; andererseits aber berichtet B a $110 \mathrm{w}$ i t $z$ (1913 b, S. 298), daß in den Rotzellen von M u 11 u s (Lipophorer mit rotem Pigment) immer nur e in Kern vorkommt. In betreff der Zellen mit rotem Lipochrom bei den Gobiiden konnte B a I l owi t z (1913 c, S. 549) nicht zu einem abschließenden Urteil über die Kernverhältnisse gelangen.

Die Le u k p horen sind vor allem durch ihren Guaninin halt gekennzeichnet. Während am Gefrierschnitt und am Totalpräparat das Guanin mehr als kleine unregelmäßige, ziemlich große Körnchen erscheint (Fig. 1, Taf. IV), zeigt es auf den Schnitten seine wahre Gestalt. Es bildet nämlich kleine, dünne Täfelchen (Fig. 4 u. 5, Taf. IV), die gruppenweise mit ihren Flächen übereinander geschichtet sind (vgl. oben B i e d e r m a n $\mathbf{n}$, der eine Andeutung dieser Verhältnisse sah). Wenn auch keine bestimmte Anordnung dieser Gruppen von Kristallplättchen besteht, so sind 
sie doch im allgemeinen so gelagert, daß ihre Fläche der Außen und der Innenwand des Bechers parallel gerichtet ist. Auf den Schnitten erscheinen sie stäbchenförmig; es handelt sich aber nicht um Nadeln, da man punktförmigen Querschnitten in entsprechender Verteilung richt begegnet. Vielmehr führt der Vergleich der Totalpräparate mit den Schnittbildern zur Ueberzeugung, daß hier kleine Plättchen vorliegen müssen.

$\mathrm{N}$ e u m a n n (1909, S. 566 f.) hat zuerst bei Amphibien deutliche Guanintäfelchen beobachtet und zwar in Guanophoren des Bauchfells vom Frosch. Ich kann für das Bauchfell der Salamander larve diese Beobachtungen durchaus bestätigen.

In den Eisenhämatoxylinpräparaten (Fig. 7 u. 8, Taf. IV) ist meist vom Guanininhalt in den Leukophoren nur eine undeutliche Streifung zurückgeblieben; der kristallinische Inhalt der Zellen ist gelöst und deshalb erscheinen sie bei dieser Färbung wesentlich heller als die Xanthophoren.

Der Kern der Guanophoren (Fig. 4 u. 8, Taf. IV) ist entsprechend der Becherform der Zelle gestaltet, also etwas abgeplattet, ferner wohl oft kleiner als der Kern der Xanthophoren und gewöhnlich etwas mehŕdem Unterrand der Zolle genähert. In einigen Fällen, aber selur viel seltener als bei den Xanthophoren, fand ich $z$ we $\mathbf{i}$ Kerne in e i ne r Leukophore (Fig. 7, Taf. IV). Zweikernige Guanophoren sind bisher wohl nirgends bekannt geworden.

Sichere Anhaltspunkte für das Vorkommen von $\mathrm{Z}$ e $\mathrm{n} \mathrm{tr}$ i o 1 e $\mathrm{n}$ in Leukophoren habe ich nicht gewinnen können.

Die bisher geschilderten Beobachtungen sind vornehnalich am Querschnitt der Haut gewonnen. Die Betrachtung von Fla $\mathrm{ch}$ $\mathrm{schnitten}$ ergänzt sie in willkommener Weise. Wie schon ältere Autoren und auch wir oben nach dem Studium des Totalpräparates berichtet haben, stoßen die „Xantholeukophoren“ als mehr oder weniger regelmäßige, polygonale, vier- bis achteckige Zellen nach Art eines einschichtigen Epithels aneinander, dessen Fläche nur von den Ausführgängen der Drüsen durchbohrt wird. Man kann schon verstehen, wie ein derartig eigentiumliches Bild bei einer weniger eingehenden Untersuchung diese Elemente als wirklich epitheliale ersclieinen lassen konnte (s. o. B i m m e r m a n n). Geht der Schnitt nahe dem Epithel durch das Niveau der Doppelzelle hindurch, so schließen die $\mathrm{X}$ a $\mathrm{nth}$ ophoren ganz dicht aneinander und sind nur durch feine Bindegewebslamellen getrennt 
(Fig. 9; Taf. IV). Hin und wieder löst sich von den Lamellen ein kleiner faden- oder lappenartiger Fortsatz ab, der in die Zellen vom Rand her einschneidet. Es handelt sich um die aufsteigenden Fasern der Kutis, welche die Doppelzellen umspinnen und, über ihnen zusammenneigend, in die kollagene Grenzlamelle übergehen. 'Auch diese ist demnach keine strukturlose Membran, auch kein Produkt des Epithels, sondern läßt stellenweise die Zusammensetzung aus abgeplatteten Bündeln erkennen. Ganz dicht unter der Grenzlamelle getroffen, erscheinen die Xanthophoren infolge der eben beschriebenen Verhältnisse weniger regelmäßig polygonal, sondern vielfach tief und unregelmäßig eingeschnitten.

War der Flachschnitt etwas tiefer, in der Höhe des Kernes der Xanthophoren, geführt, so sind diese ganz oder z.T. von einem Rahmen aus Guaninmassen, dem querdurchschnittenen Becherrand der Leukophoren, umschlossen (Fig. 9, Taf. IV). Dabei ist bemerkenswert, daß, wie im Querschnitt, auch hier keine Bindegewebslamellen zwischen der. Xanthophore und Leukophore einer Doppelzelle zu erkennen sind, so daß die beiden Bestandteile derselben einander unmittelbar berühren, durch die gemeinsame Umscheidung mit Bindegewebe aber $z \mathfrak{u}$ einer höheren Einheit verbunden erscheincn.

Kern und Plasma der Le ukophoren bieten die schon bei der Besprechung des Querschnittes hervorgehobenen Eigentümlichkeiten dar. An solchen flach getroffenen Zellen habe ich zuerst die obenerwähnten Granula gesehen (Fig. 10, Taf. IV).

Ein Flachschnitt durch die Le u k o p h or e n (Fig. 1i, Taf. IV) zeigt insofern ein vom Querschnitt abweichendes Bild, als die Zellen durch die Unterlagerung mit Melanin dunkler. aussehen, als dort. Man beachte auch, daß die Guanophoren infolge der zwischen sie eingeschobenen Ausläufer der Melanophoren keine polygonale Abflachung zeigen: sie sind rundlich begrenzt. Kern und Guaninmassen bieten bei dieser Schnittrichtung nichts Bemerkenswertes gegenüber dem Bild im Querschnitt dar.

Bis jetzt wurde nur gesagt, daß e in e Xanthophore und e i n e Leukophore zu einer Doppelzelle verbunden auftreten. Diese Regel wird von gelegentlichen Ausnahmen durchbrochen. Es finden sich nämlich auch Kombinationen, die aus $z$ we $i$ Leukophoren und e i n e $r$ Xanthophore (Fig. 5, Taf. IV) oder umgekehrt aus e i n e $r$ Leukophore und $\mathrm{zw}$ ei Xanthophoren bestehen. Man kann sich hiervon sowohl an Quer-als auch an Flachschnitten der Haut über- 
zeugen. Die letzte Art von Kombinationen läßt sich besonders leicht am Flachschnitt feststellen, weil die beiden zu, einer Kombination gehörigen Xanthophoren nicht durch Bindegewebslamellen voneinander getrennt sind, sondern von einem gemeinsamen Rahmen von Bindegewebe umfaßt werden, der sich als e in Polygon dem allgemeinen epitheloiden Mosaik einreiht.

Es erübrigt noch ein Wort über die B e z i e h unge $n$ de $r$ Melanophoren $z$ u den Doppelzellen. Nach manchen Stellen der Querschnitte (Fig. 2 u. 3, Taf. IV) könnte man den Ein druck gewinnen, daß die Melanophoren in mehrfacher Schicht übereinander gelagert sind. Das trifft aber in der Regel nicht zu. Die guirlandenartig die Doppelzellen von unten her umfassenden Melaninsicheln sind Ausläufer der etwas tiefer gelegenen Zellkörper der schwarzen Pigmentzellen (Fig. 6, Taf. IV). Im Zellkörper läßt sich der anscheinend immer nur in Einzahl vorhandene Kern ohne Bleichung des Pigmentes nur selten erkennen (Fig. 2 u. 6, Taf. IV). Jede Melanophore versorgt, wie auch aus den Untersuchungen von $B$ i e de $r$ mann und $\mathrm{Ficalbi}$ (s. o.) hervorgeht, eine $A \mathrm{nzahl}$ von Doppelzellen und man könnte in dieser Vereinigung einer Melanophore mit mehreren Doppelzellen die höchste histologische Einheit des Farbwechselorgans erblicken. -

Aus den vorstehenden Mitteilungen ergibt sich in Uebereinstimmung mit Ficalbi zweifellos, daB Xanthole ukophoren im Sinne von Gaupp beim Laubfrosch n i c h t vorkommen, daß vielmehr auch an den grünen Hautstellen Guanin und Lipochrom jedes für sich in besonderen Zellen gelegen ist. Die übereinandergeschichteten Xanthophoren (Lipophoren) und Leukophoren (Guanophoren) sind aber paarweise (seltener in anderen Kombinationen s. 0.) zu Einheiten höherer Art vereint, die so eigenartig ausgestaltet sind, daß sie wohl einen besonderen Namen verdienen. Es scheint mir am nächsten zu liegen, sie als Xanthole ukosomen zu bezeichnen; der erste Teil des Wortes soll an den alten, nunmehr aufzugebenden Namen erinnern; der zweite Teil aber lehnt sich an die von B a $110 \mathrm{witz}$ (1913 a u. c) bei Fischen angewandte Nomenklatur für die Kombinationen verschiedenartiger Farbzellen zu sogenannten , chromatischen Organen" an und soll darauf hinweisen, daB hier eine $t$ y $p$ sche Vereinigung zweierlei verschiedener Farbzellen zu einem einheitlichen Gebilde, 
e in e r Do p pelzelle, vorliegt. Man könnte diese ,chromatischen Organe" beim Laubfrosch auch Lipoguanosomen benennen, wodurch der charakteristische Inhalt der beiden Komponenten in unzweideutiger Weise angezeigt würde.

Auf Grund dieser morphologischen Befunde müssen die Vorgänge der Pigmentverschiebung derart umgedeutet werden, daß erstens eine Vermengung von Lipochrom und Guanin nicht vorkommt, daß ferner die Ballung des Lipochroms ein Vorgang ist, mit dem die Xanthophoren allein betraut sind und daß schließlich, wenn an den Guaninmassen Bewegungsvorgänge sich vollziehen sollten (s. o.), diese einzig von den Guanophoren geleistet werden. Damit fallen die oben erwähnten Schwierigkeiten bei der Verlagerung der zweierlei Pigmente fort, die sich bei ihrem Vorkommen nebeneinander in e i n e $r$ Zelle darbieten würden.

Wenn man sich fragt, ob die eigenartige Verbindung je einer Xanthophore und einer Leukophore $z u$ einem Xantholeukosom bei der Erzeugung der grünen Farbe eine besondere Rolle spielt, so ist der zunächst vielleicht nahe liegende Gedanke, daß die bikonvexe Linsenform der Xanthophore eine konzentrierende Wirkung auf das durchgehende Licht ausübe, von der Hand zu weisen, da die Masse dieser Linse durch die eingelagerten stark lichtbrechenden Lipochromkörnchen optisch inhomogen wird. Dagegen bleibt es denkbar, daß die Becherform der Le uk o$\mathrm{p}$ ho $\mathrm{re}$ insofern einen Vorteil für die Erzielung der grünen Farbe darstellt, als bei einem Lichteinfall senkrecht zur Haut die nach Art eines Hohlspiegels gelagerten Guaninmassen Strahlen nach allen Richtungen hin reflektieren werden, somit die grüne Farbe unter sehr verschiedenen Stellungen des beobachtenden Auges wahrnehmbar wird. Diese Wirkung wird auch bei schräger Beleuchtung der Haut allerdings in eingeschränktem Maße auftreten. Zum Teil ist die Existenz der typisch ausgebildeten Xantholeukosomen beim Laubfrosch ausschlaggebend für die sehr gleichmäßig grüne Farbe, welche dieses Tier an ausgedehnten Hautflächen besitzt, im Gegenteil etwa zum Wasserfrosch, bei dem die grüne Farbe über größere Hautpartien hin nicht diese sammtige Ebenmäßigkeit besitzt.

Uebrigens liegen bei $R$ a n a $f u s c$ and $R$. es $c u l$ en $t a$, ferner bei $\mathrm{B}$ uf o viridis die Dinge hinsichtlich des Vorkommens von "Xantholeukophoren" ebenso wie beim Laubfrosch: die beiden Pigmente kommen getren $n t$ in $z$ wei e r le i $Z$ elle $n$ 
vor. Allerdings sind gewisse Unterschiede gegenüber Hyla vorhanden, über die an anderer Stelle berichtet werden. soll.

\section{Literaturverzeichnis.}

B a 110 witz, E., 1913 a. Die chromatischen Organe in der Haut von Trachinus vipera Cuv. Ein Beitrag zur Kenntnis der ChromatophorenVereinigungen bei Knochenfischen. Z. f. wiss. Zool. Bd. 104, S. 471 bis 529 , Taf. $14-18$.

Derse $1 \mathrm{be}, 1913 \mathrm{~b}$. Ueber Erythrophoren in der Haut der Seebarbe, Mullus L., und über das Phänomen der momentanen Ballung und Ausbreitung ihres Pigmentes. Nach Beobachtungen an der lebenden Zelle. Arch. f. mikr. Anat. Bd. 83, S. 290-304, Taf. 15 und 16.

Derselbe, $1913 \mathrm{c}$. Ueber schwarzrote und sternförmige Farbzellkombinationen in der Haut von Gobiiden. Ein weiterer Beitrag zur Kenntnis der Chromatophoren und Chromatophoren-Vereinigungen bei Knochenfischen. Z. f. wiss. Zool. Bd. 106, S. 527-593, Taf. 8-12.

B r ü cke, E., 1852. Untersuchungen über den Farbenwechsel des afrikanischen Chamaeleons. Neudruck Leipzig 1893 in Ostwalds „Klassiker der exakten Naturwissenschaften".

B i ed e rma n n, W., 1892. Ueber den Farbenwechsel der Frösche. Pflügers Archiv Bd. 51, S. 455-508, Taf. XI.

B i $m$ m e rm a n n, E. H., 1878. Ueber den Einfluß der Nerven auf die Pigmentzellen des Frosches. Dissertation Straßburg.

Ehrmann, S., 1892. Beitrag zur Physiologie der Pigmentzellen nach Versuchen am Farbenwechsel der Amphibien. Arch. f. Derm. und Syph. Jahrg. 24, S. 519-539, Taf. XI.

F i c a l bi, E., 1896. Ricerche sulla struttura minuta della pelle degli anfibi (Pelle degli anuri della famiglia delle Hylidae). Atti della R. Accademia Peloritana in Messina, Anno 11 (1896/97) Messina, zitiert nach $v$ a n $R$ y n berk.

Fuchs, R. F., 1914. Der Farbenwechsel und die chromatische Hautfunktion der Tiere. Handbuch der vergl. Physiologie, herausgegeben von Winterstein, Bd. III, 1. Hälfte, II. Teil, S. 1189-1656.

G a u p p, E., 1904. A. Eckers und R. Wi e ders he i m s Anatomie des Frosches, 3. Abt. Braunschweig.

H e m pelm a n n, F., 1908. Der Frosch. Zugleich eine Einführung in das praktische Studium des Wirbeltierkörpers. Leipzig.

H e r i n g, Th., 1869. Ueber die Bewegungen der sternförmigen Pigmentzellen und die dadurch erzeugten Veränderungen in der Hautfarbe der Frösche, mitgeteilt von Professor $\mathrm{H}$ o y e $\mathrm{r}$ in Warschau. Zentralbl. f. d. med. Wiss. Nr. 4 , S. $49-54$.

$\mathrm{K}$ e $11 \mathrm{e} \mathrm{r}$, B., 1895. Ueber den Farbenwechsel des Chamäleons und einiger anderer Reptilien. Pflügers Archiv Bd. 61, S.' 123-168, Taf. 4. 
$\mathrm{N}$ e u $\mathrm{m}$ a $\mathrm{n}$, E., 1909. Guaninkristalle in den Interferenzzellen der Amphibien. Virch. Arch. Bd. 196, S. 566-576.

Pernitzsch, F., 1913. Zur Analyse der Rassenmerkmale der Axolotl. 1. Die Pigmentierung junger Larven. Arch. f. mikr. Anat. Bd. 82, S. 148-205, Taf. $11-13$.

P ouchet, G., 1876. Des changements de coloration sous l'influence des nerfs. Journ. de l'anat. et de la physiol. 1876, p. 1-90 et 113-165, Tab. I-IV.

R y n b e r k, G. v a n, 1906. Ueber den durch Chromatophoren bedingten Farbenwechsel der Tiere (sog. chromatische Hautfunktion). Ergebn. d. Phys. Bd. V, S. 347-571.

S c h m i d t, W. J., 1917. Die Chromatophoren der Reptilienhaut. Arch. f. mikr. Anat. Bd. 90, S. 98-259, Taf. 5-9.

Siedlecki, M., 1909. Zur Kenntnis des javanischen Flugfrosches. Biol. Zentralbl. Bd. 28, S. 704-737, Taf. VII/VIII.

Wa g n e r, K., 1911. Beiträge zur Entstehung des jugendlichen Farbkleides der Forelle (Salmo fario). Internationale Revue der gesamten Hydrobiologie und Hydrographie. Biol. Supplement Bd. 2, S. 1-32, Taf. XI.

Winkler, F, 1910 a. Beobachtungen über die Bewegungen der Pigmentzellen. Arch. f. Derm. u. Syph. Bd. 100, S. 255-260.

De r s el be, $1910 \mathrm{~b}$. Studien über Pigmentbildung. Arch. f. Entwicklungsmech. Bd. 29, S. 616-641, Taf. 22-25.

v. Wittich, 1854 a. Die grüne Farbe unserer Frösche, ihre physiologischen und pathologischen Veränderungen. Müllers Arch. Jahrg. 1854, S. $41-59$.

Derselbe, $1854 \mathrm{~b}$. Entgegnung auf Herrn Harless': Ueber die Chromatophoren des Frosches. Ebenda Jahrg. 1854, S. 257-269.

\section{Erklärung der Abbildungen.}

Alle Abbildungen beziehen sich auf $\mathrm{gr}$ ü n e $\mathrm{H}$ a utstelle $\mathrm{n}$ vom $\mathrm{L} a \mathrm{u} b \mathrm{fr}$ os ch (Hyla arborea) und sind mit Zuhilfenahme des Abbeschen Zeichenapparates hergestellt worden unter Benutzung von Zeiß' Apochromat $2 \mathrm{~mm}$ N.A. 1,30 und der Kompensationsokulare 4 und 8 . Die Vergrößerung ist 500fach bzw. 1000fach (die Zeichenfläche befand sich in Abstand von $250 \mathrm{~mm}$ von der Austrittspupille des Mikroskops).

In allen Abbildungen bedeutet

$$
\begin{aligned}
& \mathrm{X}=\text { Xanthophoren (Lipophoren), } \\
& \mathrm{L}=\text { Leukophoren (Guanophoren), } \\
& \mathrm{M}=\text { Melanophoren. }
\end{aligned}
$$

Fig. 1. Gefrierschinit durch die Haut von der Dorsalseite des oberschenkels, in $10 \%$ Formol fixiert, ungefärbt in Wasser untersucht. Nur die Chromatophoren sind 
wiedergegeben und die Lage der Epidermis (E) angedeutet. In jedem $\mathrm{X}$ antholeuk os om sind deutlich der lipochromhaltige Teil, die $X$ anthophore (X) und der guaninhaltige Teil, die Leukophore (L), zu erkennen; in drei Xantholeukosomen erscheinen die Kerne der Xanthophoren, in einem derselben außerdern die Stelle des Kernes einer Leukophore. Melanophoren $(M)$ mit stark geballtem Pigment schmiegen sich unten den Xantholeukosomen an. Zwischen den Xantholeukosomen bleiben Spalten frei, in die die hier nicht sichtbaren (weil vom Pigment entleerten) Ausläufer der Melanophoren sich hineinerstrecken. Schnittdicke $20 \mu$, Vergr. $500: 1$.

Fig. 2. Schnitt durch die $\mathrm{R}$ ü ckenhaut; die Epidermis (E) ist ganz dargestellt, von der Kutis nur der obere, die Chromatophoren enthaltende Teil. Dicht unter der Epidermis die Lage der X a n $\mathrm{t}$ h 0 $\mathrm{l}$ e u k o s o m e n; der Lipochromfarbstoff in den Xanthophoren ist durch die Präparationsmethode gelöst; die Guaninkristalle in den Leukophoren dagegen sind erhalten. Zwischen die Reihe der Xantholeukosomen ist an einer Stelle ein Bindegewebskern eingekeilt. In einer Melanophore sieht man den Zellkern. Fixierung Fle m ming Gemisch, Schnittdicke $10 \mu$, Färbung ThioninEosin, Vergrößerung $500: 1$.

Fig. 3. Schnitt durch die $R$ ü $\mathrm{c} k$ e $\mathrm{n} h \mathrm{a} u \mathrm{t}$, wie Fig. 2. In zwei Xanthophoren sind je $z$ wei Kerne sichtbar. Die Guaninkristalle der Leukophoren sind großenteils durch die Eisenhämatoxylinbehandlung (Beize!) gelöst und nur schwach zu erkennen. Fixierung Fle m ming Gemisch, Schnittdicke $10 \mu$, Färbung Eisenhämatoxylin, Vergr. $500: 1$.

Fig. 4. Xantholeukosom mit $z$ weikerniger Xanthophore. In der Leukophore sind die Guaninkristalle deutlich sichtbar. Fixierung Fle m m ings Gemisch, Schnitfdicke $10 \mu$, Färbung Thionin-Eosin, Vergr. 1000:1.

Fig. 5. Xantholeukosom, das aus einer Xanthophore und z w e i Leukophoren besteht. Fixierung, Schnittdicke, Färbung, Vergrößerung wie in Fig. 4.

Fig. 6. Eine Gruppe von $\mathrm{X}$ ant hole uk os omen, die von den Ausläufern einer $\mathrm{Mela}$ nophore versorgt wird. Fixiefung, Schnittdicke, Färbung wie in Fig. 4, Vergr. 500:1.

Fig. 7. Xantholeuk osom mit einer $\mathrm{z}$ weikernigen Leukophore, Fixierung Fle m mings Gemisch, Schnittdicke $10 \mu$, Färbung Eisenhämatoxylin, Vergr. 1000: 1 .

Fig. 8. Xantholeukosom mit zweikerniger Xanthophore, Guaninkristalle der Leukophore fast vollständig verschwunden. Fixierung, Schnittdicke, Färbung, Vergrößerung wie in Fig. 7.

Fig. 9. Flachschnitt, durch drei Xantholeukosomen in der Höhe der Xanthophoren; z. T. sind die äußersten Ränđer der 
Ueber die sog. Xantholeukophoren beim Laubfrosch.

Becher der zugehörigen Leukophoren in der Schnittebene -gelegen. Plasma der Xanthophoren ausgesprochen körnig in der Umgebung des Kernes. Fixjerung, Schnittdicke, Färbung, Vergrößerung wie in Fig. 7.

Fig. 10. Flachschnitt durch eine $\mathrm{X}$ anthoph ore, welche dicht mit kleinen Granula erfüllt ist. Fixierung, Schnittdicke, Färbung, Vergrößerung wie in Fig. 7.

Fig. 11. Flachschnitt durch fünf Xantholeukosome in der Höhe der L e Ukophoren. Zwischen den Zellen die Ausläufer der Melanophoren. Fixierung, Schnittdicke, Färbung, Vergrößerung wie in Fig. 7. 
Archiv f mikroskop. Anatomie Bd. XCIT Abt.I.

Taf. $N$.

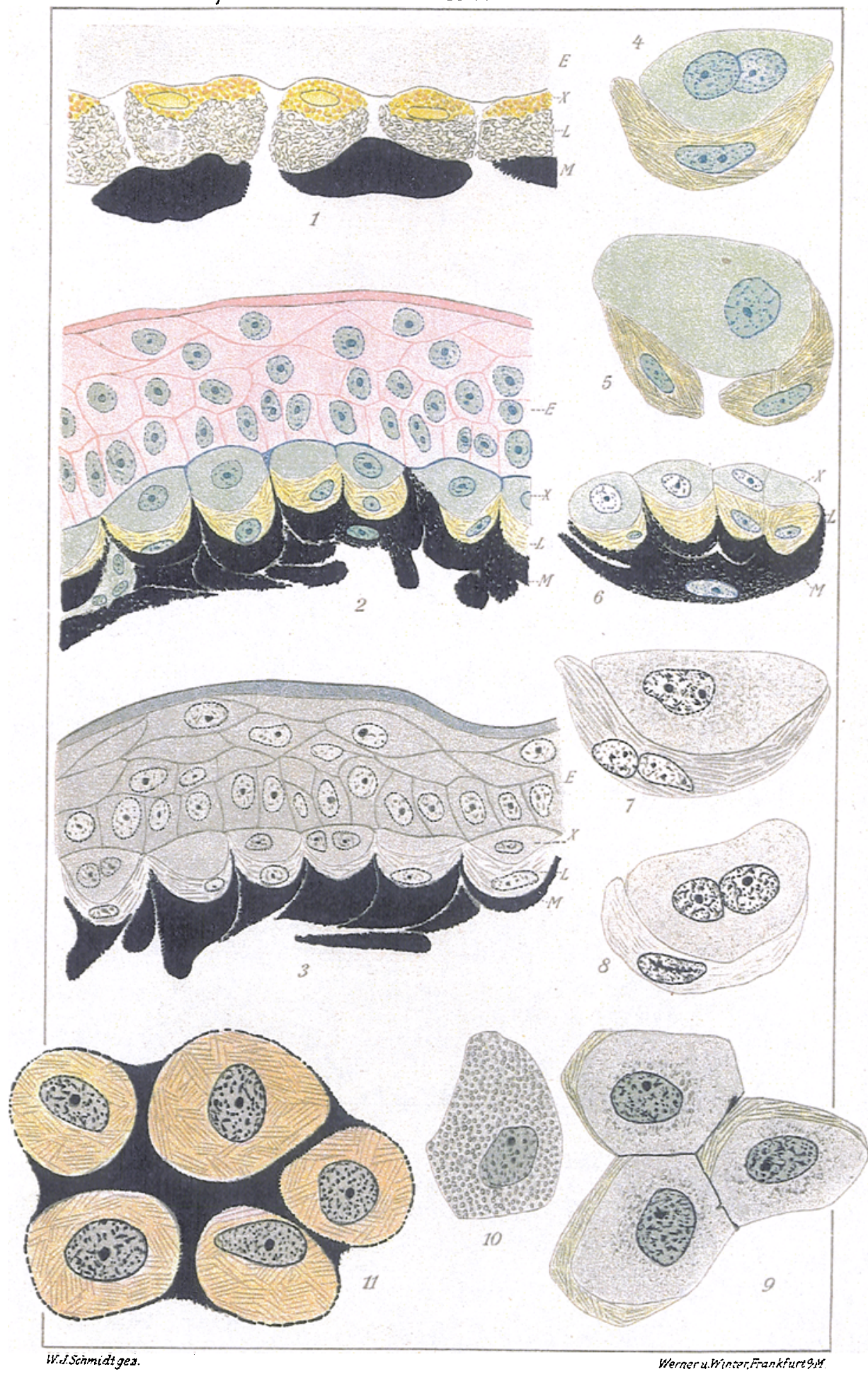

